\title{
AN INSIGHT INTO COMMON FILTERING IN NOISY SIMO BLIND SYSTEM IDENTIFICATION
}

\author{
Mark R. P. Thomas ${ }^{\dagger}$, Nikolay D. Gaubitch ${ }^{\star}$, Emanuël A. P. Habets*, Patrick. A. Naylor \\ $\dagger$ Microsoft Research, Redmond, USA. email: markth@microsoft.com \\ * Dept. of Electrical \& Electronic Engineering, Imperial College London, UK \\ email: \{ndg, p.naylor\} @imperial.ac.uk \\ * International Audio Laboratories Erlangen, University of Erlangen-Nuremberg, Germany \\ email: emanuel.habets@audiolabs-erlangen.de
}

\begin{abstract}
The effect of additive sensor noise on single-input-multiple-output (SIMO) blind system identification (BSI) algorithms based upon cross-relation (CR) error is investigated. Previous studies have shown that additive noise in the observed signal results in systems comprising the true estimated channels convolved with an erroneous 'common filter', and additionally that identification and removal of this filter significantly improves estimation error. However, the source of the common filter remained an open question. This paper explains the common filter through a first-order perturbation analysis of the CR matrix, showing that it be estimated from the perturbation and the eigenvectors of the noiseless CR matrix. The analysis given in this paper provides a new insight into the effect of noise on SIMO BSI algorithms and forms the first step towards an overall noise robust solution.
\end{abstract}

Index Terms - Blind system identification, common filtering, cross relation algorithm.

\section{INTRODUCTION}

Blind system identification (BSI) is an important analysis task for signals captured in a reverberant environment. A number of multichannel least-squares and subspace methods have been proposed that exploit the cross-relation (CR) error [1-4]. These are able to identify channels from multichannel observations if the channel order is known and identifiability conditions are satisfied [1]. When these constraints are satisfied, perfect system identification can be achieved in the absence of noise by identification of the null space of the CR matrix.

The robustness of such algorithms in the presence of additive noise is a problem that has received much attention in the literature. In the noisy case, the solution from the null space in the CR matrix is replaced by a set of solutions with small eigenvalues [3] and, with adaptive algorithms, misconvergence to an incorrect solution [5, 6]. Of particular interest is the common filter that appears in systems estimated from noisy observations, so-called because the solutions to all estimated channels are equivalent to the true channels convolved with a single, common, filter. Studies in [7-9] have shown that supervised identification and removal of the common filter improves

The authors acknowledge the financial support of the Future and Emerging Technologies (FET) programme within the Seventh Framework Programme for Research of the European Commission, under FET-Open grant number: 226007 SCENIC. Mark Thomas was at Imperial College London during the course of this work. the error in the estimated system and avoids misconvergence in the adaptive case. Other studies model the common filter as a spectral tilt in the identified systems [10] that can be removed given some $a$ priori knowledge of the magnitude spectrum of the channel.

However, the cause of the common filter remained an open question. In this paper we present a study into the effect of uncorrelated additive noise and show, by the analysis of a perturbation to the CR matrix, that noise causes a common filter to be present in all estimated channels. The analysis considers all eigenvectors of the CR matrix in the noiseless and noisy cases, establishing that all solutions with corresponding nonzero eigenvalues are related to the true system by a common filter.

The remainder of this paper is organized as follows. In Sec. 2, some background on CR-based algorithms is given. The common filtering resulting from additive noise noise is analysed in Sec. 3. An experimental example is given in Section 4 and conclusions are drawn in Sec. 5.

\section{BACKGROUND ON CR ALGORITHMS}

Consider a speech signal recorded in a noisy environment with an array of microphones. The observed signals at channel $m \in$ $\{1,2, \ldots, M\}$ are given by

$$
\begin{aligned}
& \mathbf{x}_{m}(n)=\mathbf{y}_{m}(n)+\boldsymbol{\nu}_{m}(n) \\
& \mathbf{y}_{m}(n)=\mathbf{H}_{m} \mathbf{s}(n),
\end{aligned}
$$

where $\mathbf{s}(n)=[s(n) s(n-1) \ldots s(n-2 L+1)]^{T}, \mathbf{x}_{m}(n)=$ $\left[x_{m}(n) x_{m}(n-1) \ldots x_{m}(n-L+1)\right]^{T}, \mathbf{y}_{m}(n)=\left[y_{m}(n) y_{m}(n-\right.$ 1) ... $\left.y_{m}(n-L+1)\right]^{T}, \boldsymbol{\nu}_{m}(n)=\left[\nu_{m}(n) \nu_{m}(n-1) \ldots \nu_{m}(n-\right.$ $L+1)]^{T}$ are segments of the speech signal, noisy observation, clean observation, and noise starting at sample $n$ respectively and $\mathbf{H}_{m}$ denotes the filtering matrix. The length of each segment is $L$ samples. The filtering matrix is defined by

$$
\mathbf{H}_{m}=\left[\begin{array}{cccccc}
h_{m, 0} & \ldots & h_{m, L-1} & \ldots & \ldots & 0 \\
0 & h_{m, 0} & \ldots & h_{m, L-1} & \ldots & 0 \\
\vdots & \ddots & \ddots & \ddots & \ddots & \vdots \\
0 & \ldots & \ldots & h_{m, 0} & \ldots & h_{m, L-1}
\end{array}\right],
$$

which is derived from the filtering vector $\mathbf{h}_{m}=$ $\left[\begin{array}{llll}h_{m, 0} & h_{m, 1} & \ldots & h_{m, L-1}\end{array}\right]^{T}$. Since the impulse responses are 
assumed to be slowly time-varying, $\mathbf{h}_{m}$ is independent of $n$. By concatenating all $M$ outputs of (1), a system of equations

$$
\mathbf{x}(n)=\mathbf{H s}(n)+\boldsymbol{\nu}(n)
$$

can be obtained where $\mathbf{x}(n)=\left[\mathbf{x}_{1}^{T}(n) \mathbf{x}_{2}^{T}(n) \ldots \mathbf{x}_{M}^{T}(n)\right]^{T}, \mathbf{H}=$ $\left[\begin{array}{lllll}\mathbf{H}_{1}^{T} & \mathbf{H}_{2}^{T} & \ldots & \mathbf{H}_{M}^{T}\end{array}\right]^{T}$ and $\boldsymbol{\nu}(n)=\left[\begin{array}{llll}\boldsymbol{\nu}_{1}^{T}(n) & \boldsymbol{\nu}_{2}^{T}(n) & \ldots & \boldsymbol{\nu}_{M}^{T}(n)\end{array}\right]^{T}$. The aim is to find $\mathbf{h}=\left[\begin{array}{llll}\mathbf{h}_{1}^{T} & \mathbf{h}_{2}^{T} & \ldots & \mathbf{h}_{M}^{T}\end{array}\right]^{T}$ using only $\mathbf{x}(n)$. This means that, for a given output $\mathbf{x}(n)$, a unique solution to $\mathbf{h}$ should be obtained up to a non-zero scale factor across all channels. This scale factor is irrelevant in most of acoustic signal processing applications. The identifiability conditions [1] state that the covariance matrix of $\mathbf{s}(n)$ be full rank and that there are no common zeros shared between channels; in the noiseless case $\mathbf{h}$ can then be identified exactly.

\subsection{The CR Algorithm}

From (2) the following cross-relation can be deduced [1] in the absence of noise,

$$
\begin{aligned}
x_{m} * h_{m^{\prime}} & =s * h_{m} * h_{m^{\prime}}=x_{m^{\prime}} * h_{m}, \\
& m, m^{\prime}=\{1,2, \ldots, M\}, m \neq m^{\prime},
\end{aligned}
$$

so that at time $n$,

$$
\mathbf{x}_{m}^{T}(n) \mathbf{h}_{m^{\prime}}=\mathbf{x}_{m^{\prime}}^{T}(n) \mathbf{h}_{m} .
$$

Multiplying (6) by $\mathbf{x}_{m}(n)$ and taking the expectation on both sides leads to

$$
\mathbf{R}_{x_{m} x_{m}} \mathbf{h}_{m^{\prime}}=\mathbf{R}_{x_{m} x_{m^{\prime}}} \mathbf{h}_{m},
$$

where $\mathbf{R}_{x_{m} x_{m^{\prime}}}=\mathrm{E}\left\{\mathbf{x}_{m}(n) \mathbf{x}_{m^{\prime}}^{T}(n)\right\}$ is the cross-correlation matrix between $\mathbf{x}_{m}(n)$ and $\mathbf{x}_{m^{\prime}}(n)$. The relation described by (7) results in $M(M-1)$ distinct equations. Summing (7) over $M-1$ cross-relations associated with one particular channel $\mathbf{h}_{m}$ results in

$$
\sum_{m=1, m \neq m^{\prime}}^{M} \mathbf{R}_{x_{m} x_{m}} \mathbf{h}_{m^{\prime}}=\sum_{m=1, m \neq m^{\prime}}^{M} \mathbf{R}_{x_{m} x_{m}} \mathbf{h}_{m}
$$

For $m=1, \ldots, M$ we then have a total of $M$ equations that can be expressed in matrix form as

$$
\mathbf{R h}=\mathbf{0}_{[M L \times 1]}
$$

with

$$
\mathbf{R}=\left[\begin{array}{cccc}
\sum_{m \neq 1} \mathbf{R}_{x_{m} x_{m}} & -\mathbf{R}_{x_{2} x_{1}} & \cdots & -\mathbf{R}_{x_{M} x_{1}} \\
-\mathbf{R}_{x_{1} x_{2}} & \sum_{m \neq 2} \mathbf{R}_{x_{m} x_{m}} & \cdots & -\mathbf{R}_{x_{M} x_{2}} \\
\vdots & \vdots & \ddots & \vdots \\
-\mathbf{R}_{x_{1} x_{M}} & -\mathbf{R}_{x_{2} x_{M}} & \cdots & \sum_{m \neq M} \mathbf{R}_{x_{m} x_{m}}
\end{array}\right]
$$

and $\mathbf{0}_{M L \times 1}$ is a $M L \times 1$ null vector. If the channel identifiability conditions are satisfied, the rank of $\mathbf{R}$ is $M L-1$, and the solution to $\mathbf{h}$ lies within the null space of $\mathbf{R}[1,2]$.

\section{THE EFFECT OF NOISE ON CR-BASED ALGORITHMS}

In the presence of additive noise, $\boldsymbol{\nu}_{m}(n) \neq 0 \forall m$. This section analyses the effect of noise on CR algorithms by demonstrating that a common filter is present in the solution. The equality in (9) must first be rewritten as

$$
\mathbf{R} \hat{\mathbf{h}}=\mathbf{e},
$$

where $\hat{\mathbf{h}}$ is an estimate of $\mathbf{h}$ and $\mathbf{e}$ is an error term. Recall that $\mathbf{y}(n)$ and $\mathbf{x}(n)$ are, respectively, the noiseless and noisy observations. Considering an eigenanalysis for both noisy and noiseless cases, the following relations are found:

$$
\begin{aligned}
& \mathbf{R}^{y y} \hat{\mathbf{h}}_{i}^{y y}=\lambda_{i}^{y y} \hat{\mathbf{h}}_{i}^{y y} \\
& \mathbf{R}^{x x} \hat{\mathbf{h}}_{i}^{x x}=\lambda_{i}^{x x} \hat{\mathbf{h}}_{i}^{x x},
\end{aligned}
$$

where the superscripts $y y$ and $x x$ denote the CR matrices and eigenvectors for noiseless and noisy cases respectively and the subscript $i=\{0,1, \ldots, M L-1\}$ enumerates the solution. In (12) the only solution equal to the true channel is that which corresponds to the null space of $\mathbf{R}^{y y}$ where $\lambda_{0}^{y y}=0$ and $\hat{\mathbf{h}}_{0}^{y y}=\mathbf{h}$. In the presence of noise, where only $\hat{\mathbf{h}}_{i}^{x x}$ are available, it is sensible to choose the solution that corresponds to the smallest eigenvalue as it represents a total least squares solution to (11). However, it has been noted that solving to minimize e by selection of the eigenvector corresponding to the smallest eigenvalue $\mathbf{R} \hat{\mathbf{h}}=\lambda \hat{\mathbf{h}}$ does not lead to a noise-robust solution [3].

\subsection{Common Filtering in Noiseless Solutions}

In the noiseless case, there exist $M L$ viable solutions to the eigenvalue problem $\mathbf{R h}=\lambda \mathbf{h}$. The eigenvectors $\hat{\mathbf{h}}_{i}^{y y}$ are assumed to be normalized such that they form an orthonormal basis in $\mathbb{R}^{M L}$ [11]. Let $\mathbf{H}_{i}^{\text {cyy }}$ be a filtering matrix that maps $\mathbf{h}$ to $\hat{\mathbf{h}}_{i}^{y y}$ such that

$$
\hat{\mathbf{h}}_{i}^{y y}=\mathbf{H}_{i}^{c y y} \mathbf{h},
$$

where $\mathbf{H}_{0}^{\mathrm{cyy}}=\mathbf{I}_{M L \times M L}$. Therefore, in the noiseless case, if (12) is a solution then

$$
\mathbf{R}^{y y} \mathbf{H}_{i}^{\mathrm{c} y y} \mathbf{h}=\lambda_{i}^{y y} \mathbf{H}_{i}^{\mathrm{cyy}} \mathbf{h}
$$

is also a solution. The orthogonality of the eigenvectors requires that the filtering matrix satisfies

$$
\mathbf{h}^{T}\left(\mathbf{H}_{i}^{\text {cyy }}\right)^{T} \mathbf{H}_{j}^{\text {cyy }} \mathbf{h}=0 .
$$

We will refer to $\mathbf{H}_{i}^{\mathrm{cyy}}$ as a common filtering matrix since it is applied to a stacked vector $\mathbf{h}$ containing $M$ channel responses.

\subsection{Perturbation Analysis of Noisy Solutions}

In the case of noisy solutions, $\mathbf{R}^{x x} \hat{\mathbf{h}}_{i}^{x x} \neq \mathbf{0}$. An eigenvalue perturbation analysis has been conducted in [12] where the authors investigate first- and second-order perturbations with a view to obtaining an accurate estimate of the estimated channel as a function of perturbations to $\mathbf{R}^{y y}$. In this paper we apply first-order perturbation analysis to establish that all noisy solutions are related to the true channel by a common filter $\mathbf{H}_{i}^{\mathrm{c} x x}$.

It is expected that sensor noise causes small perturbations to the clean cross-relation matrix such that [11]

$$
\begin{aligned}
\mathbf{R}^{x x} & =\mathbf{R}^{y y}+\mathbf{R}^{\delta} \\
\lambda_{i}^{x x} & =\lambda_{i}^{y y}+\lambda_{i}^{\delta} \\
\hat{\mathbf{h}}_{i}^{x x} & =\hat{\mathbf{h}}_{i}^{y y}+\hat{\mathbf{h}}_{i}^{\delta} .
\end{aligned}
$$


where $\left\{\mathbf{R}^{\delta}, \lambda_{i}^{\delta}, \hat{\mathbf{h}}_{i}^{\delta}\right\}=\mathcal{O}(\epsilon)$ and $\epsilon$ is presumed to be small. Substituting (17) into (13) and expanding gives

$$
\begin{gathered}
\left(\mathbf{R}^{y y}+\mathbf{R}^{\delta}\right)\left(\hat{\mathbf{h}}_{i}^{y y}+\hat{\mathbf{h}}_{i}^{\delta}\right)=\left(\lambda_{i}^{y y}+\lambda_{i}^{\delta}\right)\left(\hat{\mathbf{h}}_{i}^{y y}+\hat{\mathbf{h}}_{i}^{\delta}\right), \\
\mathbf{R}^{y y} \hat{\mathbf{h}}_{i}^{y y}+\mathbf{R}^{y y} \hat{\mathbf{h}}_{i}^{\delta}+\mathbf{R}^{\delta} \hat{\mathbf{h}}_{i}^{y y}+\mathbf{R}^{\delta} \hat{\mathbf{h}}_{i}^{\delta} \\
=\lambda_{i}^{y y} \hat{\mathbf{h}}_{i}^{y y}+\lambda_{i}^{y y} \hat{\mathbf{h}}_{i}^{\delta}+\lambda_{i}^{\delta} \hat{\mathbf{h}}_{i}^{y y}+\lambda_{i}^{\delta} \hat{\mathbf{h}}_{i}^{\delta} .
\end{gathered}
$$

The first terms on the LHS and RHS of (19) are necessarily equal according to (12). Assuming further that the second-order terms $\mathbf{R}^{\delta} \hat{\mathbf{h}}_{i}^{\delta}$ and $\lambda_{i}^{\delta} \hat{\mathbf{h}}_{i}^{\delta}$ are small, (19) reduces to

$$
\mathbf{R}^{y y} \hat{\mathbf{h}}_{i}^{\delta}+\mathbf{R}^{\delta} \hat{\mathbf{h}}_{i}^{y y} \simeq \lambda_{i}^{y y} \hat{\mathbf{h}}_{i}^{\delta}+\lambda_{i}^{\delta} \hat{\mathbf{h}}_{i}^{y y} .
$$

We assert in (17a) that the solutions to the clean and noisy case are related by a small perturbation $\mathbf{R}^{\delta}$. Given this and that the $\hat{\mathbf{h}}_{i}^{y y}$ form an orthonormal basis in $\mathbb{R}^{M L}$, it is assumed that small perturbations can be decomposed using the unperturbed solutions as a basis [11] such that

$$
\hat{\mathbf{h}}_{i}^{\delta}=\sum_{j=0}^{M L-1} c_{i, j} \hat{\mathbf{h}}_{j}^{y y},
$$

where $c_{i, j}$ are scalar weights. A first-order eigenvalue perturbation analysis yields a standard result for the eigenvalues of the perturbed system [11],

$$
\lambda_{i}^{\delta} \simeq \hat{\lambda}_{i}^{\delta}=\left(\hat{\mathbf{h}}_{i}^{y y}\right)^{T} \mathbf{R}^{\delta} \hat{\mathbf{h}}_{i}^{y y} .
$$

Correspondingly the approximation to (17b) is given by

$$
\hat{\lambda}_{i}^{x x}=\lambda_{i}^{y y}+\hat{\lambda}_{i}^{\delta} .
$$

Similarly the weights $c_{i, j}$ can be estimated by the standard result [11]:

$$
c_{i, j} \simeq \hat{c}_{i, j}= \begin{cases}\left(\left(\hat{\mathbf{h}}_{j}^{y y}\right)^{T} \mathbf{R}^{\delta} \hat{\mathbf{h}}_{i}^{y y}\right) /\left(\lambda_{i}^{y y}-\lambda_{j}^{y y}\right) & \text { for } i \neq j \\ 0 & \text { for } i=j .\end{cases}
$$

The noisy solutions $\hat{\mathbf{h}}_{i}^{x x}$ can therefore be approximated by substituting (21) and (24) into (17c),

$$
\begin{aligned}
\hat{\mathbf{h}}_{i}^{x x} & =\hat{\mathbf{h}}_{i}^{y y}+\hat{\mathbf{h}}_{i}^{\delta} \\
& \simeq \hat{\mathbf{h}}_{i}^{y y}+\sum_{\substack{j=0 \\
i \neq j}}^{M L-1} \frac{\left(\hat{\mathbf{h}}_{j}^{y y}\right)^{T} \mathbf{R}^{\delta} \hat{\mathbf{h}}_{i}^{y y}}{\lambda_{i}^{y y}-\lambda_{j}^{y y}} \hat{\mathbf{h}}_{j}^{y y} .
\end{aligned}
$$

A final simplification comes from defining a new set of weights,

$$
\hat{d}_{i, j}= \begin{cases}\hat{c}_{i, j} & \text { for } i \neq j \\ 1 & \text { for } i=j,\end{cases}
$$

and reformulating (25) as

$$
\hat{\mathbf{h}}_{i}^{x x} \simeq \sum_{j=0}^{M L-1} \hat{d}_{i, j} \hat{\mathbf{h}}_{j}^{y y} .
$$

Having established a closed-form expression for $\hat{\mathbf{h}}_{i}^{x x}$ in terms of $\hat{\mathbf{h}}_{i}^{y y}, \mathbf{R}^{\delta}$ and $\lambda_{i}^{y y}$, we return to the relation in (14) and substitute into (27),

$$
\hat{\mathbf{h}}_{i}^{x x} \simeq \sum_{j=0}^{M L-1} \hat{d}_{i, j} \mathbf{H}_{j}^{\mathrm{c} y y} \mathbf{h} .
$$

All solutions $\hat{\mathbf{h}}_{i}^{x x}$ are therefore related to $\mathbf{h}$ by a common filter $\mathbf{H}_{i}^{\mathrm{c} x x}=\sum_{j=0}^{M L-1} d_{i, j} \mathbf{H}_{j}^{\mathrm{c} y y}$, whose first-order approximation is given in (28). The result would be exact if the true weights $d_{i, j}$ were known.

\subsection{Limit Approximations}

It is often assumed in existing studies that the noise is spectrally white and uncorrelated with the signal such that $E\left\{\boldsymbol{\nu}(n)^{T} \mathbf{y}(n)\right\}=$ 0 ; given a sufficiently long observation time, the cross terms are assumed negligible and the noise CR matrix is simplified to $\mathbf{R}^{\delta}=$ $\sigma^{2} \mathbf{I}$ [3]. This has the property of adding $\sigma^{2} \mathbf{I}$ to the eigenvalues of $\mathbf{R}^{y y}$ while leaving the corresponding eigenvectors intact [3]. This result is consistent with (22) since when $\mathbf{R}^{\delta}=\sigma^{2} \mathbf{I}$,

$$
\lambda_{i}^{\delta}=\left(\hat{\mathbf{h}}_{i}^{y y}\right)^{T} \mathbf{R}^{\delta} \hat{\mathbf{h}}_{i}^{y y}=\sigma^{2} \forall i .
$$

Similarly the perturbation to the eigenvalues in (25) becomes,

$$
\left(\hat{\mathbf{h}}_{j}^{y y}\right)^{T} \mathbf{R}^{\delta} \hat{\mathbf{h}}_{i}^{y y}=\sigma^{2}\left(\hat{\mathbf{h}}_{j}^{y y}\right)^{T} \hat{\mathbf{h}}_{i}^{y y}=0,
$$

leading to $\hat{\mathbf{h}}_{i}^{x x}=\hat{\mathbf{h}}_{i}^{y y}$.

Although the assumption of $\mathbf{R}^{\delta}=\sigma^{2} \mathbf{I}$ provides an elegant simplification to the noise analysis, the common filtering analysis is better-suited to practical implementations of such algorithms in which a limited amount of data is available such that $\mathbf{R}^{\delta} \neq \sigma^{2} \mathbf{I}$, so the effect of noise must be considered.

\section{EXPERIMENTATION}

The following experiment demonstrates the common filter in an estimated system using noisy observations and aims to validate the closed-form estimation of the perturbed eigenvalues $\lambda_{i}^{x x}$ and coefficients $d_{i, j}$. An acoustic impulse response (AIR) was simulated using the method of images [13] for a room measuring $5 \times 6 \times 3 \mathrm{~m}$ and reverberation time $T_{60}=0.3 \mathrm{~s}$ with a linear array of 5 microphones spaced at $0.2 \mathrm{~m}$ intervals. The channels were truncated to 128 taps and a clean observation, $\mathbf{y}(n)$, was generated with a WGN source of duration $5 \mathrm{~s}$. A noisy observation, $\mathbf{x}(n)$, was created by adding WGN to the clean observation to achieve an SNR of $10 \mathrm{~dB}$. The system was analysed by finding eigenvector $\hat{\mathbf{h}}_{0}^{x x}$ corresponding to the smallest eigenvalue of $\mathbf{R}^{x x}$. The normalized projection misalignment (NPM) [14] between $\mathbf{h}$ and $\hat{\mathbf{h}}_{0}^{x x}$ was $-8.2 \mathrm{~dB}$.

Fig. 1 shows (a) the true channel $\mathbf{h}$, (b) the estimated channel from noisy observations $\hat{\mathbf{h}}_{0}^{x x}$ and (c) the first 64 taps of the estimated common filter $\mathbf{h}_{0}^{\mathrm{c} x x}$. The effect of the common filter can be observed in (b) immediately following sparse peaks in (a), for example around 130 samples. Fig. 2 (a) shows the clean eigenvalues $\lambda_{i}^{y y}$ (o), noisy eigenvalues $\lambda_{i}^{x x}(\square)$ and the eigenvalues estimated using (23), $\hat{\lambda}_{i}^{x x}$ $(\times)$. In the clean case, the smallest eigenvalue is necessarily nearzero as $\mathbf{R}^{y y}$ is known to be rank deficient. In the noisy case there are approximately 15 small eigenvalues of similar value. The clean and noisy eigenvalues differ by a constant that is approximately equal to the noise variance as predicted by (29). Fig. 2 (b) shows the decomposition coefficients of $\hat{\mathbf{h}}_{0}^{x x}$ on $\hat{\mathbf{h}}_{j}^{y y}$ (०) calculated by the inner product $d_{0, j}=\left(\hat{\mathbf{h}}_{j}^{y y}\right)^{T} \hat{\mathbf{h}}_{0}^{x x}$. The estimated decomposition coefficients, $\hat{d}_{0, j}$, were calculated with (26) $(\square)$. Coefficients beyond the 20th solution are near-zero, suggesting that the common filter can be decomposed into a function of relatively few low-order clean eigenvectors $\hat{\mathbf{h}}_{i}^{y y}$. In both (a) and (b) a close correlation is seen between the calculated and estimated values, validating the assumptions made in the perturbation analysis. Small errors can attributed to the limitation of the analysis to first order perturbations and numerical errors in estimating the eigenvalues and eigenvectors of $\mathbf{R}^{x x}$ and $\mathbf{R}^{y y}$. 


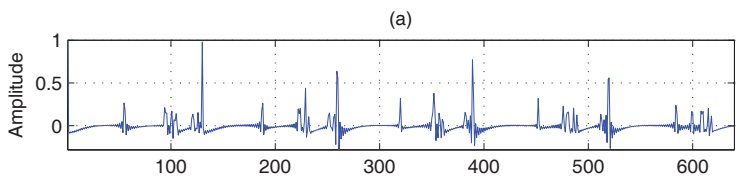

(b)

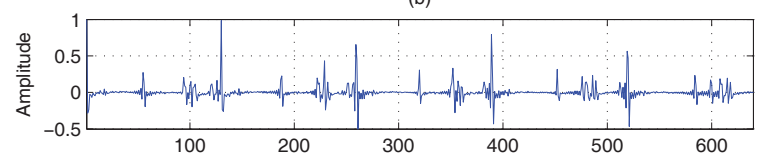

(c)

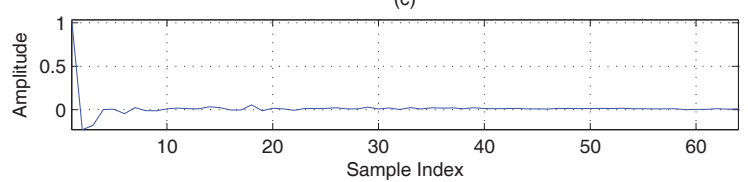

Fig. 1. (a) Simulated acoustic system $\mathbf{h}$, (b) system estimated in the presence of observation noise $\hat{\mathbf{h}}_{0}^{x x}$ and (c) the first 64 samples of the common filter $\mathbf{h}_{0}^{\mathrm{c} x x}$ that relates (a) to (b).

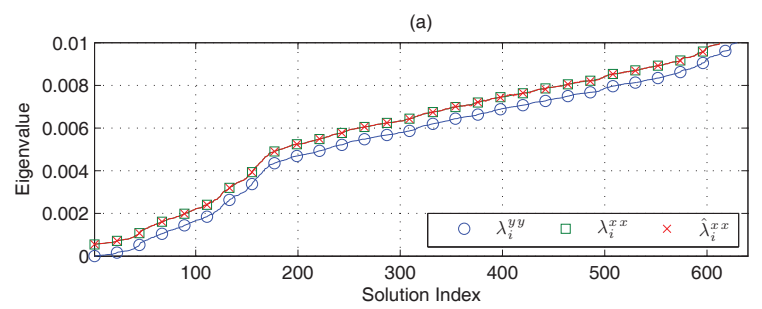

(b)

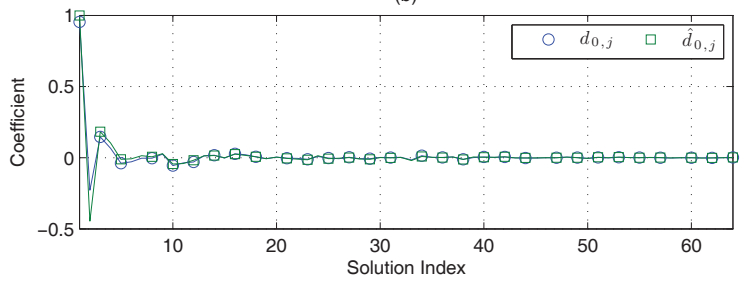

Fig. 2. (a) Eigenvalues of the noiseless CR matrix (o), noisy CR matrix ( $\square$ ) and estimated noisy eigenvalues using $(23)(\times)$, (b) coefficients estimated by $d_{0, j}=\left(\hat{\mathbf{h}}_{j}^{y y}\right)^{T} \hat{\mathbf{h}}_{0}^{x x}$ (o) and (26) ( $\square$ ). A close correlation is seen in both cases. In (a) the eigenvalues of the noisy and clean systems differ approximately by the noise variance.

\section{CONCLUSION}

The cross-relation algorithm for SIMO blind system identification has been investigated in the presence of additive noise. Previous studies have revealed, through experimentation, the presence of a common filter that relates the estimated system to the true system, however the cause remained an open question. This paper considered the effect of first-order perturbations to the cross-relation matrix and showed analytically that noise produces a common filter in the estimated system. The deleterious effects of the common filter remain problematic as a solution to its blind estimation and removal is yet to be found. However, the explanation presented in this paper provides new insight for future algorithms that exploit knowledge of the cause of common filtering.

\section{REFERENCES}

[1] G. Xu, H. Liu, L. Tong, and T. Kailath, "A least-squares approach to blind channel identification," IEEE Trans. Signal Process., vol. 43, no. 12, pp. 2982-2993, Dec. 1995.

[2] C. Avendano, J. Benesty, and D. R. Morgan, "A least squares component normalization approach to blind channel identification," in Proc. IEEE Intl. Conf. on Acoustics, Speech and Signal Processing (ICASSP), Mar. 1999, vol. 4, pp. 1797-1800.

[3] S. Gannot and M. Moonen, "Subspace methods for multimicrophone speech dereverberation," EURASIP Journal on Applied Signal Processing, vol. 2003, no. 11, pp. 1074-1090, 2003.

[4] Y. Huang and J. Benesty, "Adaptive multi-channel least mean square and Newton algorithms for blind channel identification," Signal Processing, vol. 82, pp. 1127-1138, Aug. 2002.

[5] M. K. Hasan and P. A. Naylor, "Analyzing effect of noise on LMS-type approaches to blind estimation of SIMO channels: robustness issue," in Proc. European Signal Processing Conf. (EUSIPCO), Florence, Italy, Sept. 2006.

[6] P. A. Naylor and N. D. Gaubitch, Eds., Speech Dereverberation, Springer, 2010.

[7] D. Schmid and G. Enzner, "Robust subsystems for iterative multichannel blind system identification and equalization," in IEEE Pacific Rim Conf. on Communications, Computers and Signal Processing, 2009, pp. 889-893.

[8] M. R. P. Thomas, N. D. Gaubitch, E. A. P. Habets, and P. A. Naylor, "Supervised identification and removal of common filter components in adaptive blind SIMO system identification," in Proc. Intl. Workshop Acoust. Echo Noise Control (IWAENC), Tel Aviv, Israel, Aug. 2010.

[9] D. Schmid and G. Enzner, "Evaluation of adaptive blind SIMO identificaiton in terms of a normalized filter-projection misalignment," in Proc. IEEE Intl. Conf. on Acoustics, Speech and Signal Processing (ICASSP), Prague, Czech Republic, May 2011.

[10] N. D. Gaubitch, K. Hasan, and P. A. Naylor, "Noise robust adaptive blind channel identification using spectral constraints," in Proc. IEEE Intl. Conf. on Acoustics, Speech and Signal Processing (ICASSP), Toulouse, France, May 2006, vol. 5.

[11] L. N. Trefethen and D. Bau, Numerical Linear Algebra, SIAM, 1997.

[12] Z. Xu, "Perturbation analysis for subspace decomposition with applications in subspace-based algorithms," IEEE Trans. Audio, Speech, Lang. Process., vol. 50, no. 11, pp. 2820-2830, Nov. 2002.

[13] J. B. Allen and D. A. Berkley, "Image method for efficiently simulating small-room acoustics," J. Acoust. Soc. Am., vol. 65, no. 4, pp. 943-950, Apr. 1979.

[14] D. R. Morgan, J. Benesty, and M. Sondhi, "On the evaluation of estimated impulse responses," IEEE Signal Process. Lett., vol. 5, no. 7, pp. 174-176, July 1998. 